\title{
A Parametric Analysis on the Sound Absorbing Performance of the Locally Resonant Sonic Material Using a Mass-Damper-Spring Model
}

\author{
Bo Yuan ${ }^{1, *}$, Min Jiang1, Miao He², Shuai Tang ${ }^{1}$, Li Zhang1, Minglin Tu \\ ${ }^{1}$ Department of Machinery and Electrical Engineering, Logistical Engineering University, Chongqing, China \\ ${ }^{2}$ Department of Military Engineering Management, Logistical Engineering University, Chongqing, China
}

\section{Email address:}

ramboyuanbo@aliyun.com (Bo Yuan)

*Corresponding author

\section{To cite this article:}

Bo Yuan, Min Jiang, Miao He, Shuai Tang, Li Zhang, Minglin Tu. A Parametric Analysis on the Sound Absorbing Performance of the Locally Resonant Sonic Material Using a Mass-Damper-Spring Model. American Journal of Physics and Applications.

Vol. 4, No. 5, 2016, pp. 124-133. doi: 10.11648/j.ajpa.20160405.11

Received: July 11, 2016; Accepted: July 20, 2016; Published: August 3, 2016

\begin{abstract}
The locally resonant sonic material (LRSM) is a kind of structural composite. Such composite typically consists of an elastic matrix periodically embedded with metallic spheres, which are coated with soft rubber. Owing to its capability of controlling the low frequency sound, the LRSM has a promising prospect in the application of underwater acoustic materials. This paper proposes a mass-damper-spring model to explain the sound absorbing mechanism of the LRSM, and derives analytical formulae to evaluate the absorbing performance. After reasonable simplification, the analytical formulae can intuitively illustrate the relationship between the absorbing performance and the parameters of the LRSM. The correctness of the physical model was verified by comparing the analytical evaluation with the numerical result calculated by the layer-multiple-scattering method. The result shows that the sound absorption of the LRSM is induced by the energy dissipation of the damped local resonator subjected to excitations. The influence of the parameters on the absorbing performance of the LRSM is analysed systematically. It is shown that a resonator with a heavier core and a stiffer coat can produce a better sound absorbing performance.
\end{abstract}

Keywords: Local Resonance, Sound Absorption, Underwater Acoustic Material, Mass-Damper-Spring Model

\section{Introduction}

The locally resonant sonic material (LRSM) is a kind of structural composite, which is composed of periodically or randomly arrayed local resonators as the inclusion and elastic or fluid materials as the host. The local resonators (LRs) are typically continuous structures, which possess both acoustic behaviours and resonant modes, such as Helmholtz resonators for air-borne sound [1-3] or metal cores coated with soft rubber for solid-borne sound [4-6]. Such composites are capable of controlling waves with the lattice constant two orders of magnitude smaller than the relevant wavelength [4]. This unique property gives rise to a variety of novel phenomena and applications in the area of sound and vibration [1-30]. These intriguing features include low-frequency band gaps [5, 7-11], low-frequency sound shielding [12-15], low-frequency sound absorption [16-21], negative dynamic density or elastic modulus [2, 22-25], and even negative refraction [26, 27]. On account of their negative dynamic properties and negative refraction behaviours which natural materials do not possess, LRSMs are also designated as acoustic metamaterials [23, 26, 28-30].

Although recent studies have been focusing on the negative refraction of LRSMs, there is a promising prospect in using such composite as underwater sound absorbing materials. Several papers have been devoted to investigating the potential of LRSMs in the low-frequency underwater sound absorption [16-21]. Zhao et al. analysed the sound absorbing performance of LRSMs with viscosity theoretically for the first time [16]. A following research on the absorption 
behaviour of such composite was carried out by using the Mie scattering and the multiple scattering theories [17]. Then the feasibility of low-frequency acoustic absorption of LRSMs was experimentally verified [18]. Based on the finite element method (FEM), Wen et al. analysed the effect of locally resonant modes on the absorbing performance of LRSMs [19]. The optimization of sound absorption of LRSMs had also been studied by employing genetic algorithms [20]. Moreover, a locally resonant phononic woodpile had been proposed and investigated for the underwater sound absorption [21]. However, most of these researches are based on sound field models, FEMs or experiments, which are not efficient enough for the parametric analysis, and not intuitive enough for revealing the absorbing mechanism.

Simplified mechanical models may be helpful for revealing the physical insights of the sound absorption of the LRSM. After the proposal of the LRSM [4], Goffaux et al. first applied a chain of masses and springs to explain the asymmetric peaks observed in the transmission spectra of a finite slab of coated cylinders [15]. The same method was applied to analyse the dispersion relation of their infinite counterpart [31]. Hirsekorn introduced a simpler mass-spring model in order to predict the resonant frequency of local resonators [14]. Wang et al. improved it with a better estimation of equivalent parameters and extended it to predict the lower and upper edges of resonant gaps for two and three dimensional LRSMs [9]. Recently, Huang et al. devoted to studying the double negativity, the band gap and the wave attenuation of an infinite mass-in-mass lattice model $[32,33]$. However, few studies have considered using a mechanical analogue to investigate the sound absorption performance of the LRSM.

Inspired by these forerunners' work, this paper evaluates the absorbing performance of the LRSM by using a mass-damper-spring model. Based on the long-wavelength limit, analytical formulae are derived from the proposed model, which relates the absorbing coefficient with the parameters of the LRSM. The verification of the physical model is carried out by comparing the analytical evaluation with the numerical result of the layer-multiple-scattering
(LMS) method, and the absorbing mechanism of the LRSM is clarified in detail. Furthermore, the influence of the material and the structural parameters on the absorbing performance of LRSM is analysed systematically.

\section{The Mass-Damper-Spring Model for the Evaluation of the LRSM's Absorption}

This paper focuses on the locally resonant sonic material with spherical resonators, which are periodically arrayed, in order to enable the comparison of analytical evaluation with the numerical calculation of LMS method [34]. As is known to all, local resonators (LRs) can be arranged in several different patterns [5], and the Bragg scattering effect can be stimulated in the composite if the wavelength of waves is comparable to the structural periodicities $[35,36]$. However, since this paper mainly discusses the absorption of the LRSM induced by the local resonance, which arises at the long-wavelength frequency region, we ignore the Bragg scattering effect and choose one slab of square arrayed resonators as the research subject, whose side view is shown in Fig. 1(a). In this figure, the dotted circular areas represent the cores of resonators, the white annuli around cores represent the coats of resonators and the grey hollow rectangle indicates the matrix of the LRSM. Figure 1(b) shows the diagram of a single unit cell of the slab. The radius of the core is denoted by $r_{1}$, the thickness of the coat by $h$, the lattice constant of the cell by $d$, and the thickness of the slab by $L$. Each region of the composite is assumed as an elastic material characterized by mass density $\rho_{i}$, Young's modulus $E_{i}$, and Poisson's ratio $\sigma_{i}$, with the subscript $i=1,2,3$ representing the matrix, the coat, and the core, respectively. In addition, the coat has a hysteretic damping with the loss factor denoted by $\eta_{2}$. The surrounding material is assumed to be elastic or fluid materials whose characteristic impedance is matched with the matrix, so as to avoid the reflection of the boundary affecting the absorbing performance of the composite.

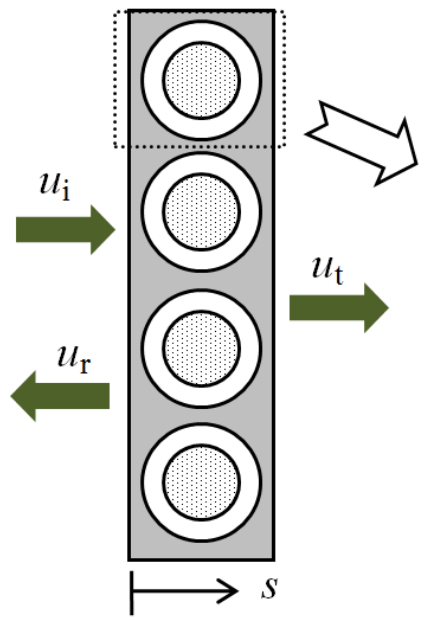

(a)

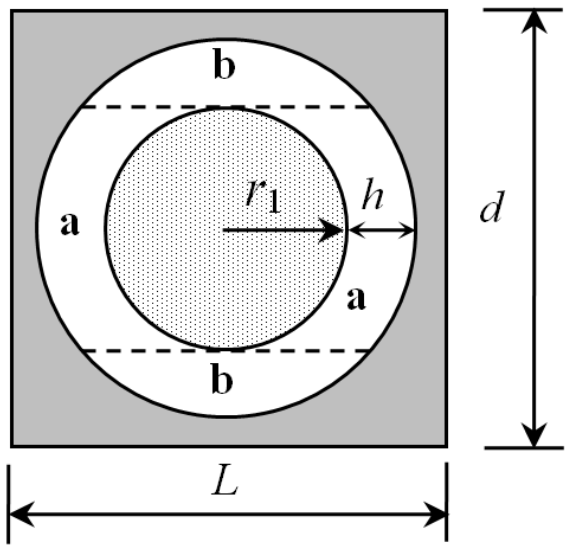

(b)

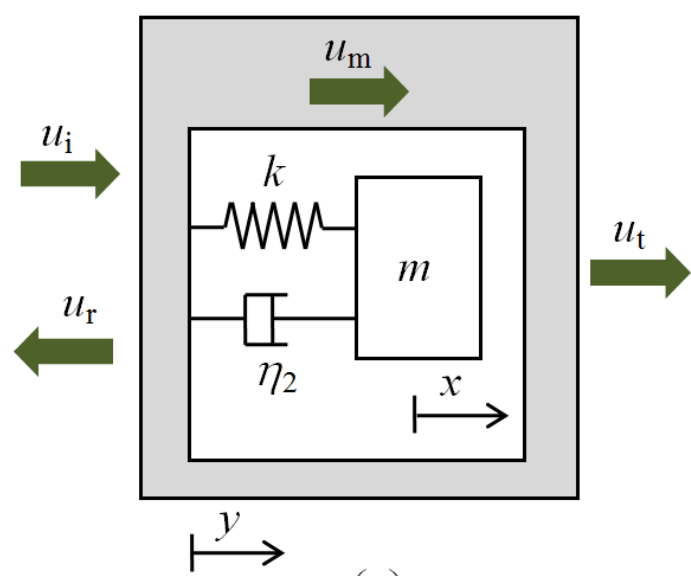

(c)

Fig. 1. (a) The side view of the LRSM considered in this paper, (b) the diagram of a single unit cell, and (c) the schematic of the physical model. 
As shown in Fig. 1(a), a plane wave is normally incident onto the left side of the composite. If the propagating direction of the incoming wave is defined as $s$-axis and the zero point is set at the left interface of the composite, the incoming wave can be expressed as $u_{\mathrm{i}}=U_{\mathrm{i}} \operatorname{expj} \omega t-\kappa s$, where $U_{\mathrm{i}}$ represents the amplitude of particle velocity, $\omega$ represents the angular frequency of the wave, and $\kappa$ represents the wavenumber of the wave in the surrounding medium. Although no reflection is stimulated at the interface since the impedance of ambience is matched with matrix, the local resonator can back scatter part of the propagating waves during the resonance. Hence, the reflected wave is assumed as $u_{\mathrm{r}}=U_{\mathrm{r}} \operatorname{expj} \omega t+\kappa s$, and the transmitted waves as $u_{\mathrm{t}}=U_{\mathrm{t}} \operatorname{exp\mathrm {j}}[\omega t-\kappa s-L]$. According to the long-wavelength limit, the displacement and velocity of particle at any point in the matrix should be approximately identical. Thus, it is reasonable to treat the matrix as a rigid body with a velocity of $u_{\mathrm{m}}=U_{\mathrm{m}} \operatorname{expj\omega t}$ (shown in Fig. 1(c)). Therefore, the displacement of the matrix can be derived

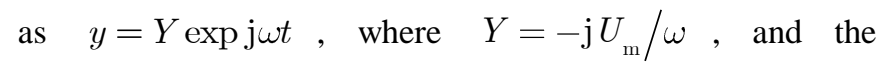
displacement of the core is expressed as $x=X \exp \mathrm{j} \omega t$.

In order to evaluate the composite's sound absorbing performance, a mass-damper-spring system with a moving foundation is proposed as the physical analogue of the LRSM, which is shown in Fig. 1(c). In the diagram, $m$ implies the equivalent mass of the resonator, $k$ the equivalent stiffness of the coat, and $\eta_{2}$ the hysteretic damping loss factor of the coat. The foundation shown as a grey box represents the rigid matrix of the LRSM. For convenience, the spring and the damper are combined as a complex stiffness $\hat{k}=k 1+\mathrm{j} \eta_{2}$, since the force induced by hysteretic damping also depends on strain [37]. According to Newton's second law, the time-independent equation of the oscillator's motion can be obtained as:

$$
-\omega^{2} m X+\hat{k} \quad X-Y=0
$$

If the normalized angular frequency is denoted by $\beta=\omega / \omega_{0}$, where $\omega_{0}=\sqrt{k / m}$ is the natural angular frequency of the corresponding lossless system, the relationship between $X$ and $Y$ can be written as

$$
X=\frac{1+\mathrm{j} \eta_{2} Y}{1-\beta^{2}+\mathrm{j} \eta_{2}}
$$

Note that $X$ is a complex value, which contains both magnitude and phase information of the oscillator's displacement. According to the dissipating behaviour of the hysteretic damping [37], the time-averaged power of the oscillator's dissipation can be derived by using Eq. (2) and $Y=-\mathrm{j} U_{\mathrm{m}} / \omega$ :

$$
\overline{\Delta W}=\pi \eta_{2} k f|X-Y|^{2}=\frac{k \eta_{2} \beta^{4}\left|U_{\mathrm{m}}\right|^{2}}{2 \omega\left[1-{\beta^{2}}^{2}+\eta_{2}^{2}\right]}
$$

However, $U_{m}$ is still unknown. By employing the continuity of velocity at the boundary of the composite and the energy conservation law, the following relations can be obtained:

$$
\left\{\begin{array}{c}
U_{\mathrm{i}}+U_{\mathrm{r}}=U_{\mathrm{m}} \\
\overline{W_{\mathrm{i}}}=\overline{W_{\mathrm{r}}}+\overline{U_{\mathrm{t}}}+\overline{\Delta W}+\overline{W_{\mathrm{t}}}
\end{array}\right.
$$

where $\overline{W_{\mathrm{i}}}, \overline{W_{\mathrm{r}}}, \overline{W_{\mathrm{t}}}$ represent the time-averaged sound power of the incoming wave, the reflected wave and the transmitted wave within one unit cell respectively. By referring the acoustic intensity formula [38], one can readily obtain $\bar{W}_{l}=\frac{1}{2} Z_{0} d^{2}\left|U_{l}\right|^{2}$, in which the subscript $l=\mathrm{i}, \mathrm{r}, \mathrm{t}$ denotes different waves, $d$ is the lattice constant of the cell, and $Z_{0}$ represents the characteristic impedance of the surrounding medium. By inserting them into Eq. (4), one can obtain $\left|U_{\mathrm{m}}\right|=2\left|U_{\mathrm{i}}\right| / Q+2$, in which

$$
Q=\frac{k \eta \beta^{4}}{\omega Z_{0} d^{2}\left[1-{\beta^{2}}^{2}+\eta_{2}^{2}\right]}
$$

Hence, the absorbing coefficient of a unit cell of the LRSM (the energy ratio of the dissipation versus the incoming wave) can be written as

$$
A=\frac{\overline{\Delta W}}{\overline{W_{\mathrm{i}}}}=\frac{k \eta \beta^{4}}{\omega Z_{0} d^{2}\left[1-{\beta^{2}}^{2}+\eta_{2}^{2}\right]} \cdot \frac{\left|U_{\mathrm{m}}\right|^{2}}{\left|U_{\mathrm{i}}\right|^{2}}=\frac{4 Q}{Q+2^{2}}
$$

To evaluate the absorbing performance of LRs by Eq. (6), the equivalent mass $(m)$ and stiffness $(k)$ of the system still need to be estimated, which depend on the deformation field of the resonator. As for the spherical LRs stimulated by normally incident waves, Wang et al. proposed three methods to estimate these parameters [9]. Specifically, method I regarded the mass of the core as the oscillator's mass, and treated the coat in region a (shown in Fig. 1(b)) as two layers of rubber with equal thickness $h$ for simplicity. Thus, the equivalent mass and stiffness can be expressed as

$$
m_{1}=\frac{4}{3} \rho_{3} \pi r_{1}^{3}, k_{1}=\frac{2 C_{11} \pi r_{1}^{2}}{h},
$$


where $C_{11}=\frac{1-\sigma_{2} E_{2}}{1-2 \sigma_{2} \quad 1+\sigma_{2}}$ is the P-wave modulus of the improved the estimation by taking account of the coat's mass and the variation of the coat's thickness in region a, whose expressions are written as:

coat, and $\rho_{3}$ is the density of the core. Moreover, method III

$$
m_{2}=m_{\mathrm{c}}+m_{\mathrm{a}} \frac{\alpha}{1+\alpha}, k_{2}=\frac{4 \pi C_{11}}{3 r_{2}^{2}-r_{1}^{2}}\left[r_{2}^{3}+r_{1}^{3}-r_{2}^{2}-r_{1}^{2^{3 / 2}}\right] .
$$

In the above formula, $\alpha=\frac{m_{\mathrm{a}}+m_{\mathrm{b}}+m_{\mathrm{m}}}{m_{\mathrm{a}}+m_{\mathrm{c}}}$ denotes the mass ratio between the foundation and the oscillator, $m_{\mathrm{a}}=\frac{4}{3} \rho_{2} \pi r_{2}^{3}-r_{1}^{3}-m_{\mathrm{b}} \quad$ and $\quad m_{\mathrm{b}}=\frac{4}{3} \rho_{2} \pi r_{2}^{2}-{r_{1}^{2}}^{3 / 2}$ represent the mass of the coat in region a and $\mathrm{b}$ respectively, while $m_{\mathrm{c}}=\frac{4}{3} \rho_{3} \pi r_{1}^{3}$ and $m_{\mathrm{m}}=\rho_{1}\left(L d^{2}-\frac{4}{3} \pi r_{2}^{3}\right)$ indicate the mass of the core and the matrix in one cell, where $r_{2}=r_{1}+h$ is the outer radius of the resonator.

To verify the methods proposed by Ref. [9], the forced response of the core is also calculated using a displacement field model proposed by Liu et al. [22], which can be used to retrieve the equivalent stiffness of the coat. In this approach, the magnitude of the core's displacement is written in the form of

$$
X_{\mathrm{C}}=-\frac{r_{2} g \omega}{r_{1} R \omega-\rho_{3} / \rho_{2}} Y_{\mathrm{C}}
$$

where $Y_{\mathrm{C}}$ is the magnitude of the matrix's displacement, $\omega$ represents the angular frequency of the forced vibration, $\rho_{2}$ and $\rho_{3}$ are densities of the coat and the core respectively, while $g \omega$ and $R \omega$ are two frequency-dependent functions whose expressions can be found in Ref. [22]. By substituting Eq. (9) into (1) and choosing $m_{2}$ as the mass of oscillator, one can obtain the equivalent stiffness of the coat as follow:

$$
k_{\mathrm{C}}=m_{2} \omega^{2} X_{\mathrm{C}} / X_{\mathrm{C}}-Y_{\mathrm{C}} .
$$

Additionally, before discussing the influence of parameters, the criteria also needs to be decided which are used for assessing the absorbing performance of the LRSM. Since the absorption spectrum of the local resonance has a profile analogous to the frequency response of the magnitude for a damped oscillator, the peak absorption and the bandwidth of half-peak are employed to assess the absorbing performance [37]. The peak absorption, denoted as $A_{\max }$, records the maximum absorption of the LRSM which usually occurs around the resonant frequency. Correspondingly, $f_{\max }$ records the accurate frequency at the peak absorption. The bandwidth of half-peak, denoted as $\Delta f_{\text {half }}$, describes the frequency range of the spectrum where the absorbing coefficient decreases from the maximum to the half-peak value.

According to these criteria, the corresponding analytical formulae can be further deduced. However, Eq. (6) is too complicated for an intuitive analysis. If we use $\left|U_{\mathrm{m}}\right|=\left|U_{\mathrm{i}}\right|$ instead of $\left|U_{\mathrm{m}}\right|=2\left|U_{\mathrm{i}}\right| /(Q+2)$ during the derivation of Eq. (6), the absorbing coefficient will have the same expression as Eq. (5). This implies that $Q$ demonstrates the absorbing performance of the composite without considering the back scattering waves of the resonator, whereas Eq. (6) is a revised formula after including the reflection. Although $Q$ will overestimate the absorbing performance of the LRSM since the displacement of the foundation is larger than the counterpart after considering reflection, it is simpler than Eq. (6) and suitable for qualitative analysis. By substituting $m_{1}$ and $k_{1}$ into $Q$, one can obtain the expression of absorbing coefficient at the resonant frequency ( $\beta=1, \omega=\omega_{0}$ ) without including the reflection:

$$
Q_{0}=\frac{2 \pi}{\eta_{2} Z_{0} d^{2}} \sqrt{\frac{2 \rho_{3} E_{2} r_{1}^{5} 1-\sigma_{2}}{3 h 1+\sigma_{2} 1-2 \sigma_{2}}}
$$

Moreover, the damped resonant frequency can be derived from Eq. (1):

$$
f_{d}=\frac{1}{4 \pi} \sqrt{\frac{3 E_{2} 1-\sigma_{2} 1+\sqrt{1+\eta_{2}^{2}}}{\rho_{3} h r_{1} 1+\sigma_{2} 1-2 \sigma_{2}}} .
$$

Based on Eq. (5) and (11), the half-peak bandwidth can be further obtained by solving the following equation:

$$
\frac{k \eta_{2} \beta^{4}}{\omega Z_{0} d^{2}\left[1-{\beta^{2}}^{2}+\eta_{2}^{2}\right]}=\frac{\sqrt{k m}}{2 \eta_{2} Z_{0} d^{2}}
$$

It should be noted that the term $\omega$ on the left side renders Eq. (13) as a quartic polynomial equation, which is complicated and needs further simplification [39]. Since the relative angular frequencies $(\beta)$ of the half-peak edges are usually close to 1 , it is reasonable to substitute $\omega$ with $\omega_{0}$ in Eq. (13), which will lead to a quadratic polynomial equation of $\beta^{2}$. Meanwhile, if we only consider the absolute displacement of the oscillator $(X)$ when calculating its dissipating power (refer to Eq. (3)), the approximate value of the half-peak bandwidth can be obtained as 


$$
\Delta f_{\text {half }}^{\prime}=\frac{\omega_{0}}{2 \pi} \sqrt{1+\eta_{2}}-\sqrt{1-\eta_{2}}
$$

\section{Parametric Analysis on the Absorbing Performance of the LRSM}

In order to verify the correctness of the physical model, an initial case is proposed here, which has a similar configuration with Ref. [4]. The resonator is composed of a lead sphere with a radius $\left(r_{1}\right)$ of $5 \mathrm{~mm}$, coated with silicone rubber whose thickness $(h)$ is $2.5 \mathrm{~mm}$ (shown in Fig. 1(b)). The LRs are arranged in a square lattice with spatial periodicity $(d)$ and the thickness of the slab $(L)$ to be $15.5 \mathrm{~mm}$. The material parameters used are $\rho_{1}=1180 \mathrm{kgm}^{-3}$, $E_{1}=4.449 \times 10^{9} \mathrm{~Pa} \quad, \quad \sigma_{1}=0.399 \quad$ for epoxy; $\rho_{2}=1300 \mathrm{kgm}^{-3}, E_{2}=1.175 \times 10^{5} \mathrm{~Pa}, \sigma_{2}=0.469$ for silicone rubber; and $\rho_{3}=11600 \mathrm{kgm}^{-3}$,
$E_{3}=4.082 \times 10^{10} \mathrm{~Pa}, \sigma_{3}=0.370$ for lead. Besides, the silicone rubber has a hysteretic damping with $\eta_{2}=0.1$. By substituting these parameters into Eq. (7), (8), (10) and then Eq. (5) or (6), one can calculate the absorption spectra from different methods, which are shown in Fig. 2(a). The $x$-axis of the figure represents the frequency $(f)$, while the $y$-axis represents the absorbing coefficient $(A)$. As shown in the legend, the dot-dash curve demonstrates the evaluation by Eq. (5) and (7), which is denoted as $Q m_{1}, k_{1}$; the dotted curve indicates the evaluation by Eq. (6) and (8), which is named as $A m_{2}, k_{2}$; the dashed curve illustrates the evaluation by Eq.

(6) and (10), which is designated as $A m_{2}, k_{\mathrm{C}}$; while the solid curve shows the actual absorption spectrum calculated from LMS method [34]. Figure 2(b, c) shows the relative magnitude $(|X / Y|)$ and the phase angle $(\operatorname{Arg} X / Y)$ of the core versus the matrix, which are predicted by $A m_{2}, k_{\mathrm{C}}$ method.

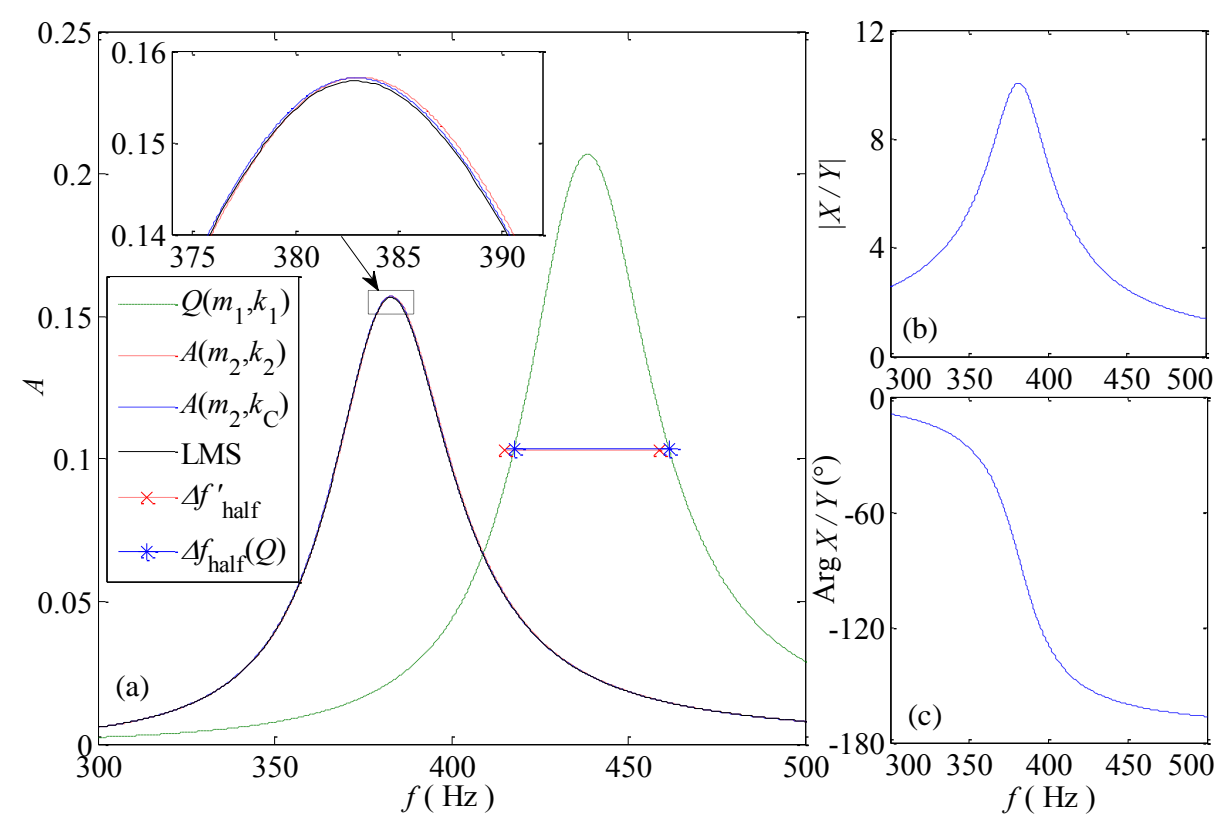

Fig. 2. (colour online) The absorption spectra (a) predicted by different methods; the relative magnitude $(b)$ and the phase angle (c) of the core against the matrix predicted by $A m_{2}, k_{\mathrm{C}}$.

In Fig. 2(a), an obvious discrepancy can be found between the absorption evaluated by $Q m_{1}, k_{1}$ and the numerical result calculated by LMS method. In fact, the overestimation of resonant frequency is caused by the excessive simplification of $m_{1}$ and $k_{1}$, and the overestimation of the maximum absorption is due to the negligence of reflected waves. In contrast, $A m_{2}, k_{2}$ and $A m_{2}, k_{\mathrm{C}}$ precisely predict the absorbing performance of the LRSM. Slight differences with LMS result can only be found in the magnified subplot. Such agreement not only verifies the correctness of the mass-damper-spring model in predicting the absorption behaviour of the LRSM, but also affirms the absorbing mechanism as the energy dissipation of the damped local resonator subjected to excitations. More explicitly, the matrix, stimulated by the incoming wave, reciprocates as a rigid body along the wave's traveling direction according to the long-wavelength limit. The metallic core which is attached to the matrix through the viscoelastic rubber will also oscillate constrainedly, whose response depends on the nature of the resonator and the characteristic of the excitation. Therefore, the relative magnitude $(|X / Y|)$ and the phase angle ( $\operatorname{Arg} X / Y$ ) of the two objects vary with the frequency of the incoming wave, as shown in Fig. 2(b, c). Since the core and the matrix never vibrate in the same way, the rubber coat will deform cyclically, and thus dissipate the energy due to its hysteretic property. Such dissipation will reach the peak when the frequency of incoming wave equals to the damped 
resonant frequency of the local resonator, because the relative magnitude will also reach its maximum. Finally, since all the cells of the LRSM have the same configuration, the absorbing performance of the composite will be exactly the same as that of a unit cell.

In addition, Fig. 2 also shows the half-peak bandwidth predicted by Eq. (11) and (14), as well as the real half-peak of $Q m_{1}, k_{1}$. The former is depicted by a dotted line segment with crosses on both ends (denoted as $\Delta f_{\text {half }}^{\prime}$ ), while the latter by a solid line segment with asterisks on the ends (denoted as $\Delta f_{\text {half }} Q$ ). Although a slight deviation on frequency can be found between the predictive and the actual values, the widths of half-peak bands are nearly the same, which implies the simplification of Eq. (14) is reasonable. The deviation is induced by the derivation of $Q_{0}$, in which the undamped natural frequency $\left(\omega_{0}\right)$ is used, whereas the real peak absorption of a damped system usually occurs at a higher frequency.

Figure 3 shows the regularity of the composite's absorption affected by the Poisson's ratio of the coat $\left(\sigma_{2}\right)$ based on the initial case. The subplot (a) presents the grey scale image of

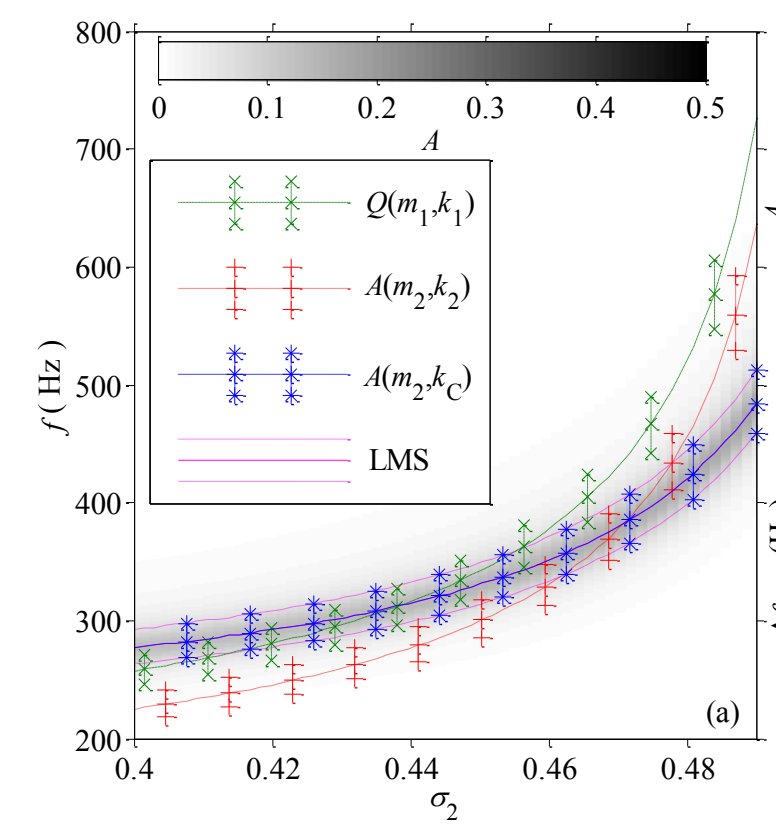

the absorption calculated by LMS method. Each point in the image indicates an absorbing coefficient of the composite at a specific $f$ and $\sigma_{2}$, where $f$ denotes the frequency of the incident wave. For comparison, the peak frequencies $\left(f_{\max }\right)$ and the edges of the half-peak calculated by $Q m_{1}, k_{1}$, $A m_{2}, k_{2}, A m_{2}, k_{\mathrm{C}}$ and LMS methods are also marked on the image. The legend in Fig. 3(a) shows the notation for different methods, e.g. the dotted curve with plus signs represents $f_{\max }$ evaluated by $A m_{2}, k_{2}$, while stems along the curve ended with plus signs indicate the edges of the half-peak. Besides, subplots $(\mathrm{b}, \mathrm{c})$ show the variation of peak absorption $\left(A_{\max }\right)$ and half-peak bandwidth $\left(\Delta f_{\text {half }}\right)$ calculated by different methods, which share the same $x$-axis $\left(\sigma_{2}\right)$ and the legend. The notation of different methods inherits the convention from Fig. 2. It needs to be noted that $f_{\max }, A_{\max }$ and $\Delta f_{\text {half }}$ of $Q m_{1}, k_{1}$ method shown in the following figures are directly estimated by Eq. (11), (12) and (14).
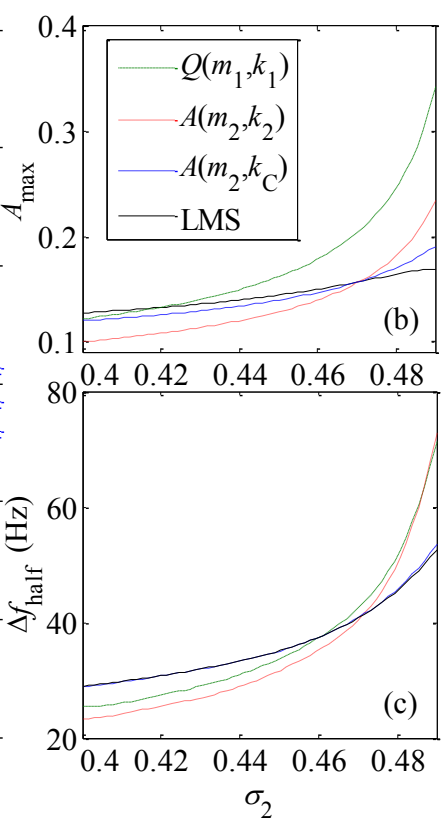

Fig. 3. (colour online) The influence of the Poisson's ratio of the coat ( $\left.\sigma_{2}\right)$ on the absorbing performance of the LRSM based on the initial case. Subplot (a) shows the grey scale image of absorption (A) calculated by LMS method and the peak frequencies with half-peak edges predicted by different methods; while subplots $(b)$ and $(c)$ show the peak absorption $\left(A_{\max }\right)$ and the half-peak bandwidth $\left(\Delta f_{\text {half }}\right)$ evaluated by different methods.

In Fig. 3(a), the peak frequencies predicted by $Q m_{1}, k_{1}$ and $A m_{2}, k_{2}$ scarcely match with LMS result except at certain circumstances ( $\sigma_{2}=0.436$ for $Q m_{1}, k_{1}$, while $\sigma_{2}=0.470$ for $A m_{2}, k_{2}$ ). Accordingly, discrepancies are also found in the peak absorption and the half-peak bandwidth of both methods, which are shown in Fig. 3(b, c). A detailed investigation ascribes the failures of $Q m_{1}, k_{1}$ and $A m_{2}, k_{2}$ methods to the inaccurate estimation of equivalent parameters. Specifically, both Eq. (7) and (8) introduce the P-wave modulus $\left(C_{11}\right)$ to calculate the equivalent stiffness of the coat [9]. Such modulus is defined as the ratio of axial stress to axial strain in a uniaxial strain state. However, this is not the case how the coat deforms. Actually, when the coat is 
stretched (or compressed) in the direction of core's vibration, it will also produce a contraction (or expansion) in the lateral direction. Since $C_{11}$ will increase rapidly when $\sigma_{2}$ approaches 0.5 , both $k_{1}$ and $k_{2}$ will be overestimated, which results that $f_{\max }, A_{\max }$ and $\Delta f_{\text {half }}$ evaluated by $Q m_{1}, k_{1}$ and $A m_{2}, k_{2}$ are significantly higher than the real. Conversely, when the coat is away from 'rubbery state' $\left(\sigma_{2}<0.42\right), k_{1}$ and $k_{2}$ will be underestimated as they ignore the stress produced by the coat in region $b$ (shown in Fig. 1(b)), thus eventually causing the evaluations of two methods lower than the real.

In contrast, $A m_{2}, k_{\mathrm{C}}$ can accurately predict the peak frequency with the varying of $\sigma_{2}$, which is shown in Fig. 3(a), since $k_{\mathrm{C}}$ is retrieved from the displacement field model. The
$A_{\text {max }}$ and $\Delta f_{\text {half }}$ evaluated by $A m_{2}, k_{\mathrm{C}}$ are also improved in comparison with other methods. The presence of a small amount of error for $A_{\max }$ is due to the estimation of $m_{2}$. Although $m_{2}$ has taken the coat's mass into account, it is still unable to describe the change of the coat's dynamic mass induced by the lateral deformation, which is related to $\sigma_{2}$.

Figures 4 to 8 shows successively the regularity of the composite's absorption based on the initial case and affected by the radius $\left(r_{1}\right)$ and the density $\left(\rho_{3}\right)$ of the core, as well as the thickness $(h)$, the Young's modulus $\left(E_{2}\right)$, and the loss factor $\left(\eta_{2}\right)$ of the coat. The meaning of the curves in each subplot is identical with that of the counterparts in Fig. 3.
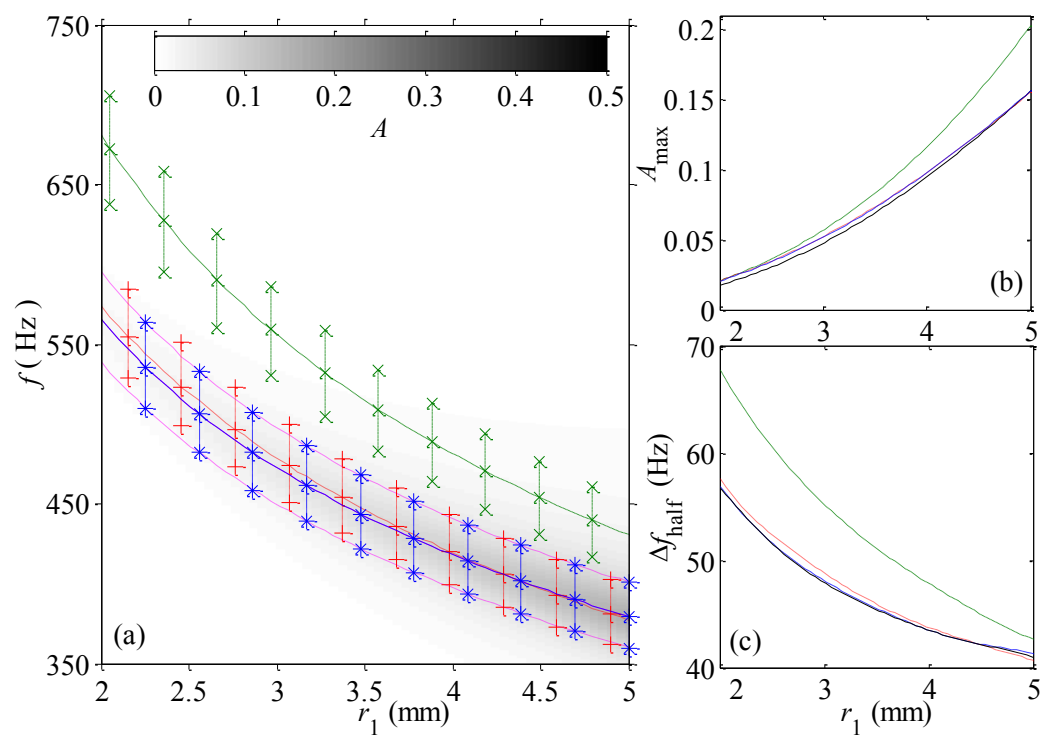

Fig. 4. (colour online) The influence of the core's radius $\left(r_{1}\right)$ on the absorbing performance of the LRSM.
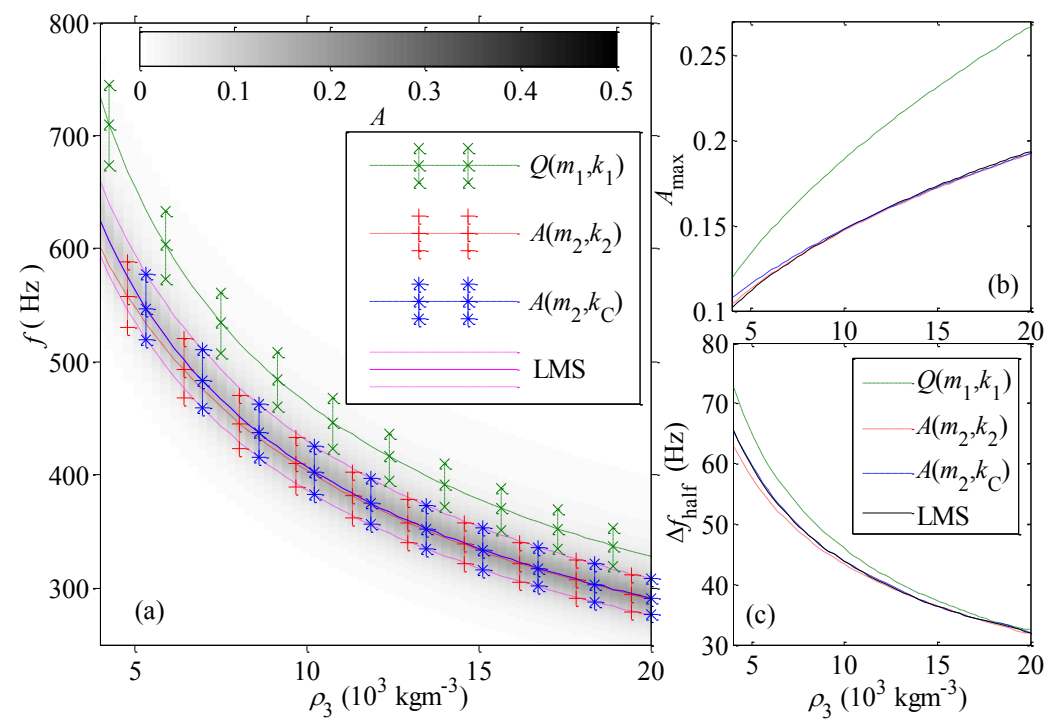

Fig. 5. (colour online) The influence of the core's density $\left(\rho_{3}\right)$ on the absorbing performance of the LRSM. 

Resonant Sonic Material Using a Mass-Damper-Spring Model
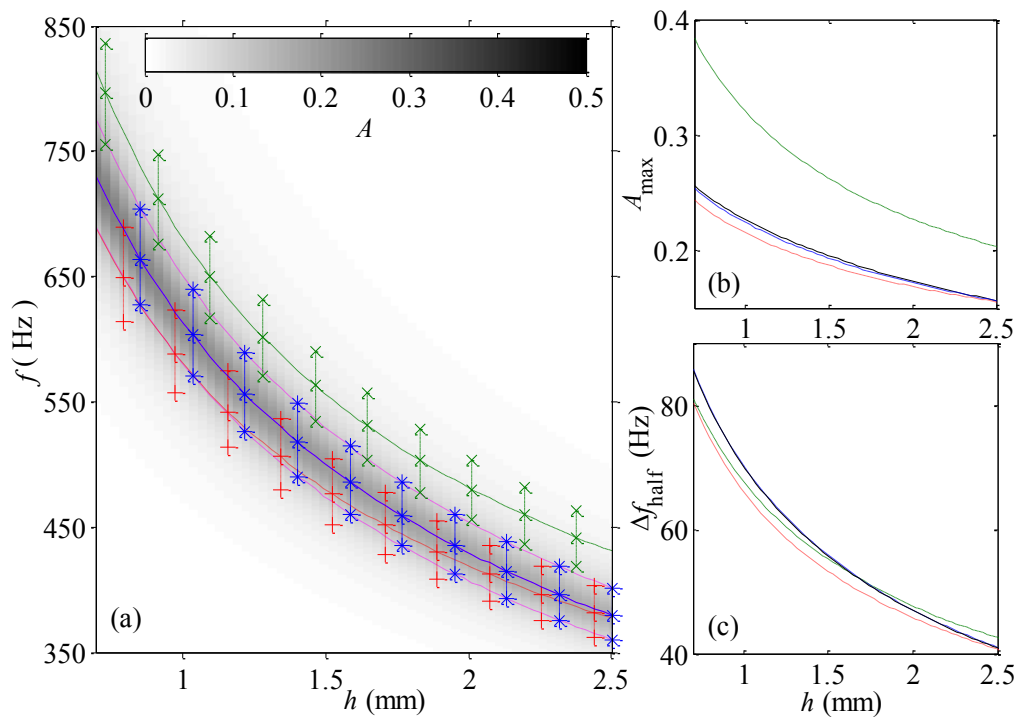

Fig. 6. (colour online) The influence of the coat's thickness ( $h$ ) on the absorbing performance of the LRSM.
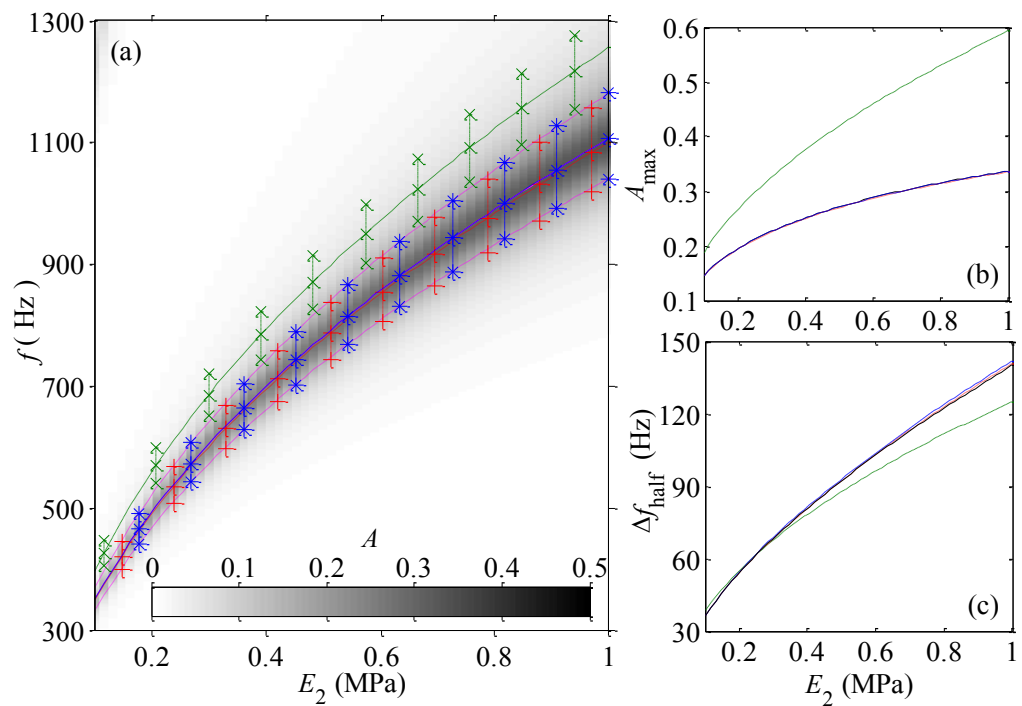

Fig. 7. (colour online) The influence of the Young's modulus of the coat $\left(E_{2}\right)$ on the absorbing performance of the LRSM.
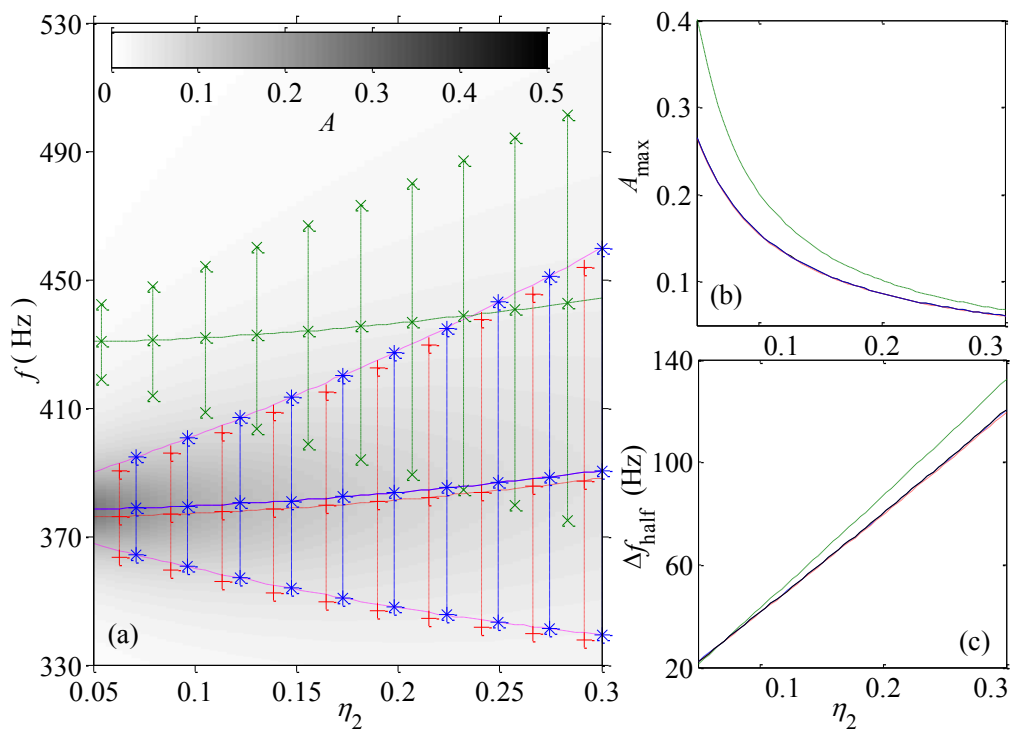

Fig. 8. (colour online) The influence of the coat's loss factor $\left(\eta_{2}\right)$ on the absorbing performance of the LRSM. 
Through a comprehensive comparison from Fig. 4 to 8 , it can be observed that the $f_{\max }, A_{\max }$ and $\Delta f_{\text {half }}$ evaluated by $A\left(m_{2}, k_{2}\right)$ are in reasonable agreement with the numerical result of LMS method, and $A\left(m_{2}, k_{\mathrm{C}}\right)$ method performs even better especially in the prediction of peak frequency. This result reinforces the correctness of the physical model, and clarifies its adaptability. Apparently, $A\left(m_{2}, k_{\mathrm{C}}\right)$ can accurately predict various sorts of LRSMs if only their matrix match with the surrounding and satisfy the long-wavelength limit, while $A\left(m_{2}, k_{2}\right)$ behaves alike except for the variation of the Poisson's ratio of coat. It can also be seen that both $f_{\max }$ and $A_{\max }$ predicted by $Q\left(m_{1}, k_{1}\right)$ are always higher than the result calculated by LMS method. These deviations are consistent with the previous analysis about Fig. 2, which is due to the overestimation of equivalent parameters and the ignorance of reflected waves. The $\Delta f_{\text {half }}$ predicted by $Q\left(m_{1}, k_{1}\right)$ also have appreciable discrepancies by comparing with actual results.

However, the accuracy of evaluation is not the purpose of deducing $Q_{0}, f_{\mathrm{d}}$ and $\Delta f_{\text {half }}^{\prime}$ (refer to Eq. (11), (12) and (14)), but the general tendency of variation. Despite the deviation, $Q\left(m_{1}, k_{1}\right)$ correctly predicts the regularities of $f_{\max }$ and $A_{\max }$ regardless of the variation of parameters. According to Eq. (11) and (12), it can be found that the density of the core $\left(\rho_{3}\right)$, the radius of the core $\left(r_{1}\right)$, and the loss factor of coat $\left(\eta_{2}\right)$ play different roles in $f_{\mathrm{d}}$ and $Q_{0}$, i.e. act as numerator and denominator in different formulae. This implies that increasing the density or the radius of the core, which also means increasing the mass of the core, will simultaneously lower the natural frequency of resonator and improve its peak absorption; decreasing $\eta_{2}$ will improve the peak absorption and decrease the damped resonant frequency as well, but the variation of $f_{\mathrm{d}}$ is much smaller than the influence by other parameters. In contrast, increasing the stiffness of coat, such as increasing the Young's modulus $\left(E_{2}\right)$ and the Poisson's ratio $\left(\sigma_{2}\right)$ of coat, or decreasing its thickness $(h)$ will increase both $f_{\mathrm{d}}$ and $Q_{0}$. All these inferences from Eq. (11) and (12) can be certified by the numerical results of LMS method as shown in Fig. 3 to 8 . On the other hand, the regularity of $\Delta f_{\text {half }}$ calculated by LMS method also coincides with the expression of Eq. (14), that either raising the resonant frequency of resonator or increasing the loss factor of coat will broaden the half-peak bandwidth.

In addition, the lattice constant $(d)$ and the characteristic impedance of the surrounding $\left(Z_{0}\right)$ shown in Eq. (11) demonstrate the proportion of dissipating power against the incoming power of one unit cell. Since they are irrelevant to the dissipating feature of the resonator, we do not show their comparisons of different methods any further, but simply explain their meanings. Apparently, with the increasing of lattice constant, more waves will propagate through the cell while the excitation of resonator remains the same, which will eventually reduce the absorbing performance of the LRSM. Similarly, if the acoustic intensity of the incoming wave remains the same, the matrix and ambient media with higher characteristic impedance will result in a smaller excitation of resonator, and so that a poorer absorption.

\section{Conclusions}

In this paper, a mass-damper-spring model is proposed to evaluate the absorbing behaviour of the LRSM. Based on the long-wavelength limit, the analytical formulae were derived from the physical model, which are used to predict the composite's absorption. By comparing the analytical model with the layer-multiple-scattering method, we validated the correctness of the physical model and revealed the absorbing mechanism of the LR as the energy dissipation of the damped local resonator subjected to excitations. A systematic discussion was carried out about the influence of material and structural parameters on the absorbing performance of LRSM. The result shows that a heavier and stiffer resonator can produce a better sound absorbing performance. Hence, the measures for optimizing the absorbing performance of the LRSM include: (i) using cores with a heavier density and larger radius, which can simultaneously reduce the natural frequency and enhance the peak absorption; (ii) using coats with a stiffer and more rubbery material with a thinner thickness to enhance the composite's absorbing performance; (iii) the loss factor of coats should be chosen moderately to balance the peak value and the bandwidth of the resonant absorption; (iv) local resonators should be arrayed closely so as to increase the energy ratio of dissipation to excitation. Finally, it is worth to be noted that such physical model is potential for evaluating the absorption behavior of other local resonators with different shapes, since it does not rely on the shape of the local resonator.

\section{Acknowledgement}

This research is partially funded by the National Natural Science Foundation of China under Grant No. 11504427 and by the Youth Foundation of the Logistical Engineering University under Grant No. YQ14-421001.

\section{References}

[1] X. Hu, C. T. Chan, J. Zi, Two-dimensional sonic crystals with Helmholtz resonators, Physical Review E 71 (2005) 055601.

[2] N. Fang, D. Xi, J. Xu, M. Ambati, W. Strituravanich, C. Sun, X. Zhang, Ultrasonic metamaterials with negative modulus, Nature Materials 5 (2006) 452-456.

[3] Y. Cheng, J. Y. Xu, X. J. Liu, One-dimensional structured ultrasonic metamaterials with simultaneously negative dynamic density and modulus, Physical Review B 77 (2008) 045134. 
[4] Z. Liu, X. Zhang, Y. Mao, Y. Y. Zhu, Z. Yang, C. T. Chan, P. Sheng, Locally resonant sonic materials, Science 289 (2000) 1734-1736.

[5] Z. Liu, C. T. Chan, P. Sheng, Three-component elastic wave band-gap material, Physical Review B 65 (2002) 165116.

[6] P. Sheng, X. X. Zhang, Z. Liu, C. T. Chan, Locally resonant sonic materials, Physica B 338 (2003) 201-205.

[7] H. Zhao, Y. Liu, G. Wang, J. Wen, D. Yu, X. Han, X. Wen, Resonance modes and gap formation in a two-dimensional solid phononic crystal, Physical Review B 72 (2005) 012301.

[8] G. Wang, X. Wen, J. Wen, L. Shao, Y. Liu, Two-dimensional locally resonant phononic crystals with binary structures, Physical Review Letter 93 (2004) 154302.

[9] G. Wang, L. Shao, Y. Liu, J. Wen, Accurate evaluation of lowest band gaps in ternary locally resonant phononic crystals, Chinese Physics 15 (2006) 1843-1848.

[10] R. Sainidou, B. Djafari-Rouhani, Y. Pennec, J. O. Vasseur, Locally resonant phononic crystals made of hollow spheres or cylinders, Physical Review B 73 (2006) 024302.

[11] Y. W. Gu, X. D. Luo, M. H. R, Optimization of the local resonant sonic material by tuning the shape of the resonator, Journal of Physics D: Applied Physics 41 (2008) 205402.

[12] K. M. Ho, C. K. Cheng, Z. Yang, X. X. Zhang, P. Sheng, Broadband locally resonant sonic shields, Applied Physics Letters 83 (2003) 5566-5568.

[13] M. Hirsekorn, P. P. Delsanto, N. K. Batra, P. Matic, Modelling and simulation of acoustic wave propagation in locally resonant sonic materials, Ultrasonics 42 (2004) 231-235.

[14] M. Hirsekorn, Small-size sonic crystals with strong attenuation bands in the audible frequency range, Applied Physics Letters 84 (2004) 3364-3366.

[15] C. Goffaux, J. Sanchez-Dehesa, A. L. Yeyati, P. Lambin, A. Khelif, J. O. Vasseur, B. Djafari-Rouhani, Evidence of Fano-like interference phenomena in locally resonant materials, Physical Review Letter 88 (2002) 225502.

[16] H. Zhao, Y. Liu, J. Wen, D. Yu, G. Wang, X. Wen, Sound absorption of locally resonant sonic materials, Chinese Physics Letters 23 (2006) 2132-2134.

[17] H. Zhao, Y. Liu, J. Wen, D. Yu, X. Wen, Tri-component phononic crystals for underwater anechoic coatings, Physics Letters A 367 (2007) 224-232.

[18] H. Zhao, J. Wen, D. Yu, X. Wen, Low-frequency acoustic absorption of localized resonances: Experiment and theory, Journal of Applied Physics 107 (2010) 023519.

[19] J. Wen, H. Zhao, L. Lv, B. Yuan, G. Wang, X. Wen, Effects of locally resonant modes on underwater sound absorption in viscoelastic materials, Journal of the Acoustical Society of America 130 (2011) 1201-1208.

[20] H. Meng, J. Wen, H. Zhao, X. Wen, Optimization of locally resonant acoustic metamaterials on underwater sound absorption characteristics, Journal of Sound and Vibration 331 (2012) 4406-4416.

[21] H. Jiang, Y. Wang, M. Zhang, Y. Hu, D. Lan, Y. Zhang, B. Wei, Locally resonant phononic woodpile: A wide band anomalous underwater acoustic absorbing material, Applied Physics Letters 95 (2009) 104101.
[22] Z. Liu, C. T. Chan, P. Sheng, Analytic model of phononic crystals with local resonances, Physical Review B 71 (2005) 014103.

[23] P. Sheng, J. Mei, Z. Liu, W. Wen, Dynamic mass density and acoustic metamaterials, Physica B 394 (2007) 256-261.

[24] S. H. Lee, C. M. Park, Y. M. Seo, Z. G. Wang, C. K. Kim, Acoustic metamaterial with negative modulus, Journal of Physics: Condensed Matter 21 (2009) 175704.

[25] S. H. Lee, C. M. Park, Y. M. Seo, Z. G. Wang, C. K. Kim, Acoustic metamaterial with negative density, Physics Letters A 373 (2009) 4464-4469.

[26] S. Guenneau, A. Movchan, PéturssonG, S. A. Ramakrishna, Acoustic metamaterials for sound focusing and confinement, New Journal of Physics 9 (2007) 399.

[27] J. Li, Z. Liu, C. Qiu, Negative refraction imaging of acoustic waves by a two-dimensional three-component phononic crystal, Physical Review B 73 (2006) 054302.

[28] L. Fok, M. Ambati, X. Zhang, Acoustic metamaterials, MRS Bulletin 33 (2008) 931-934.

[29] S. Zhang, L. Yin, N. Fang, Focusing ultrasound with an acoustic metamaterial network., Physical Review Letter 102 (2009) 194301.

[30] M. Ambati, N. Fang, C. Sun, X. Zhang, Surface resonant states and superlensing in acoustic metamaterials, Physical Review $B$ 75 (2007) 195447.

[31] C. Goffaux, J. Sanchez-Dehesa, Two-dimensional phononic crystals studied using a variational method: Application to lattices of locally resonant materials, Physical Review B 67 (2003) 144301.

[32] H. H. Huang, C. T. Sun, Wave attenuation mechanism in an acoustic metamaterial with negative effective mass density, New Journal of Physics 11 (2009) 013003.

[33] H. H. Huang, C. T. Sun, G. L. Huang, On the negative effective mass density in acoustic metamaterials, International Journal of Engineering Science 47 (2009) 610-617.

[34] R. Sainidou, N. Stefanou, I. E. Psarobas, A. Modinos, A layer-multiple-scattering method for phononic crystals and heterostructures of such, Computer Physics Communications 166 (2005) 197-240.

[35] Y. Xiao, J. Wen, X. Wen, Flexural wave band gaps in locally resonant thin plates with periodically attached spring-mass resonators, Journal of Physics D: Applied Physics 45 (2012) 195401.

[36] Y. Xiao, J. Wen, X. Wen, Longitudinal wave band gaps in metamaterial-based elastic rods containing multi-degree-of-freedom resonators, New Journal of Physics 14 (2012) 033042.

[37] C. F. Beards, Engineering Vibration Analysis with Application to Vontrol Systems, Edward Arnold, London, 1995.

[38] L. E. Kinsler, A. R. Frey, A. B. Coppens, J. V. Sanders, Fundamentals of Acoustics, 4th ed., John Wiley \& Sons, New York, 2000.

[39] D. Zwillinger, Standard Mathematical Tables and Formulae, 31st ed., CRC Press, New York, 2003. 